\title{
Evaluation of the incidence of rare diseases: difficulties and uncertainties, the example of chronic thromboembolic pulmonary hypertension
}

\author{
Gérald Simonneau ${ }^{1}$ and Marius M. Hoeper ${ }^{2}$
}

Affiliations: ${ }^{1}$ Assistance Publique-Hôpitaux de Paris, Service de Pneumologie, Hôpital Bicêtre and Hôpital Marie Lannelongue, Paris Sud University, Paris, France. ${ }^{2}$ Department of Respiratory Medicine and German Centre of Lung Research (DZL), Hannover Medical School, Hannover, Germany.

Correspondence: Gérald Simonneau, Assistance Publique-Hôpitaux de Paris, Service de Pneumologie, Hôpital Bicêtre, Hôpital Marie Lannelongue, Paris Sud University, Paris, 94275, France.

E-mail: gerald.simonneaudamail.com

@ERSpublications

The meta-analysis by Ende-Verhaar et al. provides important information and helps guide clinical practice in CTEPH http://ow.ly/Are8307RTPx

Cite this article as: Simonneau G, Hoeper MM. Evaluation of the incidence of rare diseases: difficulties and uncertainties, the example of chronic thromboembolic pulmonary hypertension. Eur Respir J 2017; 49: 1602522 [https://doi.org/10.1183/13993003.02522-2016].

Chronic thromboembolic pulmonary hypertension (CTEPH) is a rare form of pulmonary hypertension, classified as group 4 in the present clinical classification $[1,2]$. CTEPH is generally considered a late complication of one or multiple episodes of acute pulmonary embolism that have not resolved despite at least 3 months of therapeutic anticoagulation. Haemodynamically, CTEPH is characterised by a mean pulmonary arterial pressure of at least $25 \mathrm{mmHg}$ at rest, measured during right heart catheterisation.

The mechanisms of pulmonary hypertension in CTEPH are not unequivocal. Obstruction of pulmonary arteries by nonresolving thromboembolic material is generally considered the initial trigger, at least in most cases. In addition, some patients develop a secondary peripheral vasculopathy, in particular with long disease duration $[3,4]$. Histologically, these vascular lesions resemble those seen in pulmonary arterial hypertension $[5,6]$. The progressive development of these microvascular changes could explain why patients with CTEPH deteriorate if left untreated, even in the absence of recurrent pulmonary emboli.

The incidence of CTEPH in the general population is largely unknown and the same is true for the incidence of CTEPH in survivors of pulmonary embolism. The reported frequency of CTEPH after pulmonary embolism is highly variable, ranging from $0.1 \%$ to more than $10 \%$. It would be important for clinicians to have more accurate data on the incidence of CTEPH in order to provide evidence-based recommendations regarding the usefulness of screening for CTEPH in patients who have survived pulmonary embolism. In addition, more reliable data on the incidence of CTEPH after acute pulmonary embolism could allow a better estimate of the incidence of CTEPH in the general population.

In the present issue of the ERJ, ENDE-VERHAar et al. [7] present a meta-analysis of the published data on the incidence of CTEPH after pulmonary embolism. The authors selected 16 studies based on strict criteria, including prospective cohorts of patients who received a diagnosis of pulmonary embolism based on established diagnostic tools (multidetector computed tomography or ventilation/perfusion

Received: Dec 222016 | Accepted: Dec 232016

Conflict of interest: Disclosures can be found alongside this article at erj.ersjournals.com

Copyright @ERS 2017 
scintigraphy), a mean follow-up time was approximately 2 years and a diagnosis of CTEPH based on standard criteria, including right heart catheterisation. The overall weighted pooled incidence of CTEPH across all studies, totalling more than 4000 patients with a confirmed acute pulmonary embolism, was 2.3\%. In addition, the authors evaluated the incidence of CTEPH in two main predefined cohorts: "all comers" including all consecutive patients with a pulmonary embolism without any exclusion criteria; and "survivors", i.e. patients who survived for at least 6 months after pulmonary embolism.

The "all comers" population included only 2 studies (1186 patients who had been followed for 2-3 years) and the pooled CTEPH incidence in this cohort of patients was low $(0.56 \%)$. In contrast, in the "survivors" population ( $\mathrm{n}=999$, coming from different publications than the "all comers"), the pooled CTEPH incidence was 3.2\%. The risk of CTEPH was higher in patients with non-provoked pulmonary embolism than in patients with provoked pulmonary embolism and was also higher in patients with recurrent thromboembolic events than in patients with a single episode of pulmonary embolism.

The authors concluded that the $0.56 \%$ incidence in "all comers" probably provides the best reflection of the incidence of CTEPH after acute pulmonary embolism but that the $\sim 3 \%$ incidence in survivors may be more relevant for clinical practice. However, what is of most concern is the $\sim 6$-fold difference between the two cohorts. The authors provide several possible explanations but the main reason for the observed difference may be the simple fact that different studies were considered. In addition, the lower incidence in "all comers" could reflect an underestimation as patients may have died before confirmation of the CTEPH diagnosis. For example in the study by MiniaTi et al. [8], of the 834 patients included, 271 patients died with a majority of in-hospital deaths (60\% of all deaths).

At the same time, the incidence of CTEPH could have been overestimated in the "survivors" group, due to the possibility that some patients diagnosed at baseline as having acute pulmonary embolism in fact already had CTEPH. So far, only one study has properly addressed this issue; in the study by GuERIN et al. [9], a total of 146 patients with acute pulmonary embolism and no history of severe cardiac and respiratory disease were treated with therapeutic anticoagulation. During a median follow-up of 26 months, eight of the 146 patients had suspected CTEPH because of persistent dyspnoea and abnormal echocardiographic findings. CTEPH was confirmed by right heart catheterisation in seven patients $(4.8 \%$; 95\% CI 2.3-9.6). However, at the time of index acute pulmonary embolism episode, only two patients had systolic pulmonary artery pressure (sPAP) $<50 \mathrm{mmHg}$; in the remaining five patients, sPAP ranged from 62-102 $\mathrm{mmHg}$, making it highly likely that these patients were already suffering from CTEPH when a diagnosis of pulmonary embolism was made; this level of sPAP is indeed not compatible with a first episode of acute pulmonary embolism in patients without a history of cardiorespiratory diseases because the nonadapted right ventricle cannot generate such high pressures [4].

It is therefore likely that in this cohort, CTEPH was already present at the time of index acute pulmonary embolism in five out of seven patients. This notion was confirmed by review of the initial multidetector computed tomography by a senior radiologist, which showed that all patients with confirmed CTEPH had fulfilled at least two criteria for this condition at the initial presentation. Hence, the cumulative incidence of CTEPH after acute pulmonary embolism in the study by GUERIN et al. [9] was not $4.8 \%$ but $1.5 \%$ (two out of 146), at most.

In France, it has been established that about 30000 episodes of acute pulmonary embolism occur each year [10]. Considering a CTEPH incidence of $0.5 \%$ after an acute pulmonary embolism, the annual incidence of CTEPH would be 150; this would be an underestimation as 150 new CTEPH patient are operated upon each year, representing less than $50 \%$ of the overall incident CTEPH population (operated and non-operated). However, an incidence of $3 \%$ would probably be an overestimation, resulting in 900 new CTEPH patients a year in France. In the French pulmonary hypertension registry, the number of CTEPH patients newly identified each year is approximatively 300, although this registry is not totally exhaustive. Hence, the most realistic estimate of the incidence of CTEPH after acute PE is probably between 1-2\%.

The main methodological limitations in all previous studies were the small sample sizes (only two of the 16 studies included in the meta-analysis of ENDE-VerHAAR et al. [7] selected more than 500 patients with pulmonary embolism) and low numbers of CTEPH patients identified (fewer than 10 in 14 of the 16 studies). Hence, the error margin is high. Ideally, such studies should include at least 1000patients, in order to provide reliable data, such as the currently ongoing Follow-up after acute pulmonary embolism (FOCUS) study (German Clinical Trials Registry identifier DRKS00005939) [11].

Pending further data, the meta-analysis by ENDE-VerhaA et al. [7] provides important information and helps in guiding clinical practice. Given the low incidence of CTEPH after acute pulmonary embolism $(0.5-3 \%)$, it does not seem suitable to systematically screen survivors of acute pulmonary embolism for the development of CTEPH, reinforcing the recommendations made in current guidelines. Still, CTEPH 
should be considered and the threshold for further diagnostic tests should remain low in patients who remain symptomatic after one or more thromboembolic events.

\section{References}

1 Simonneau G, Gatzoulis MA, Adatia I, et al. Updated clinical classification of pulmonary hypertension. J Am Coll Cardiol 2013; 62 (25 Suppl): D34-D41.

2 Galiè N, Humbert M, Vachiery JL, et al. 2015 ESC/ERS Guidelines for the diagnosis and treatment of pulmonary hypertension. Eur Respir J 2015; 46: 903-975.

3 Lang IM, Madani M. Update on chronic thromboembolic pulmonary hypertension. Circulation 2014; 130: 508-518.

4 Hoeper MM, Madani MM, Nakanishi N, et al. Chronic thromboembolic pulmonary hypertension. Lancet Respir Med 2014; 2: 573-582

5 Moser KM, Bloor CM. Pulmonary vascular lesions occurring in patients with chronic major vessel thromboembolic pulmonary hypertension. Chest 1993; 103: 685-692.

6 Dorfmuller P, Gunther S, Ghigna MR, et al. Microvascular disease in chronic thromboembolic pulmonary hypertension: a role for pulmonary veins and systemic vasculature. Eur Respir J 2014; 44: 1275-1288.

7 Ende-Verhaar YM, Cannegieter SC, Vonk Noordegraaf A, et al. Incidence of chronic thromboembolic pulmonary hypertension after acute pulmonary embolism: a contemporary view of the published literature. Eur Respir J 2017; 49: 1601792.

8 Miniati M, Monti S, Bottai M, et al. Survival and restoration of pulmonary perfusion in a long-term fllow-up of patients after acute pulmonary embolism. Medicine 2006; 85: 253-262.

9 Guerin L, Couturaud F, Parent F, et al. Prevalence of chronic thromboembolic pulmonary hypertension after acute pulmonary embolism. Thromb Haemost 2014; 112: 598-605.

10 Oger E. Incidence of venous thromboembolism: a community-based study in Western France. EPI- GETBP Study Group. Groupe d'Etude de la Thrombose de Bretagne Occidentale. Thromb Haemost 2000; 83: 657-660.

11 Konstantinides SV, Barco S, Rosenkranz S, et al. Late outcomes after acute pulmonary embolism: rationale and design of FOCUS, a prospective observational multicenter cohort study. J Thromb Thrombolysis 2016; 42: 600-609. 